\title{
A New Developed GIHS-BT-SFIM Fusion Method Based On Edge and Class Data
}

\author{
S. Dehnavi ${ }^{1}$, A. Mohammadzadeh ${ }^{1}$
}

${ }^{1}$ Faculty of Geodesy and Geomatics, K.N.Toosi University of Technology, Tehran, Iran.

dehnavi.kntu@gmail.com

almoh2@gmail.com

KEY WORDS: GIHS_BT_SFIM, Fusion, Edge adaptive, Class based

\begin{abstract}
The objective of image fusion (or sometimes pan sharpening) is to produce a single image containing the best aspects of the source images. Some desirable aspects are high spatial resolution and high spectral resolution. With the development of space borne imaging sensors, a unified image fusion approach suitable for all employed imaging sources becomes necessary. Among various image fusion methods, intensity-hue-saturation (IHS) and Brovey Transforms (BT) can quickly merge huge amounts of imagery. However they often face color distortion problems with fused images. The SFIM fusion is one of the most frequently employed approaches in practice to control the tradeoff between the spatial and spectral information. In addition it preserves more spectral information but suffer more spatial information loss. Its effectiveness is heavily depends on the filter design. In this work, two modifications were tested to improve the spectral quality of the images and also investigating class-based fusion results. First, a Generalized Intensity-Hue-Saturation (GIHS), Brovey Transform (BT) and smoothing-filter based intensity modulation (SFIM) approach was implemented. This kind of algorithm has shown computational advantages among other fusion methods like wavelet, and can be extended to different number of bands as in literature discussed. The used IHS-BT-SFIM algorithm incorporates IHS, IHS-BT, BT, BT-SFIM and SFIM methods by two adjustable parameters. Second, a method was proposed to plus edge information in previous GIHS_BT_SFIM and edge enhancement by panchromatic image. Adding panchromatic data to images had no much improvement. Third, an edge adaptive GIHS_BT_SFIM was proposed to enforce fidelity away from the edges. Using MS image off edges has shown spectral improvement in some fusion methods. Fourth, a class based fusion was tested, which tests different coefficients for each method due to its class. The best parameters for vegetated areas was $\mathrm{k} 1=0.6, \mathrm{k} 2=0.8$; and for urban region it was $\mathrm{k} 1=0.4, \mathrm{k} 2=0.4$. Results might be useful for future studies on fusion methods and their generalization.
\end{abstract}

\section{INTRODUCTION}

$\mathrm{I}$ $\mathrm{N}$ RECENT years, the number of spectral bands in a satellite image has increased significantly. Additionally, the spatial resolution of the image has also been enhanced. For remote sensing applications, the satellite images which can simultaneously offer high spatial and spectral resolutions are often required. It means that the ground and spectrum details must be accurately presented by a single image (Tu, 2012). The benefit of merged image has been demonstrated in many practical applications, especially for vegetation, land-use, precision farming, and urban studies (Tu, 2001). Due to this, various methods for image fusion have been described earlier (Amolins, 2007; Tu, 2001, 2004, 2005, 2012; Duo, 2007; Sh. Li, 2002; Z. Li,2005; Ling, 2007; Pajares, 2004; Yang, 2007; Rahmani, 2010; Chu, 2008). Among various image fusion methods, intensity-hue-saturation (IHS) and Brovey Transforms (BT) can quickly merge huge amounts of imagery and simultaneously preserving most of the spatial information provided that the color distortion is mitigated in the fusion; as they often face color distortion problems with fused images (Tu, 2005).

Furthermore, IHS-like and BT-like approaches are indeed efficient fusion methods since they require only arithmetical operations without any statistical analysis or filter design. The Smoothing-filter-based intensity modulation (SFIM) fusion is one of the most frequently employed approaches in practice to control the trade of between the spatial and spectral information. However, SFIM is not as efficient as BT-like and IHS-like methods in the fusion of high resolution images. In addition it preserves more spectral information but suffer more spatial information loss. Its effectiveness is heavily depends on the filter design (Tu, 2012). GIHS-BT-SFIM is an approach which was proposed by $\mathrm{Tu}$ et al. as an adjustable pansharpening approach for high resolution satellite imageries. This method has shown computational advantages among other fusion methods like wavelet, and could be extended to different number of bands. The introduced IHS-BTSFIM algorithm incorporates IHS, IHS-BT, BT, BT-SFIM and SFIM methods by two adjustable parameters. On the other hand Rahmani et al. proposed an adaptive HIS Pansharpening method, whereby the edges of panchromatic image combine with the multispectral image to increase the spatial information as well as preserving the spectral quality. Another method is to incorporate edge information on GIHS_BT_SFIM fused image. An improvement is proposed in this study to combine previous approaches and test the results. Furthermore, a class-based image fusion method is introduced in this work to choose the best parameters for each class. 


\section{GIHS-BT-SFIM}

\subsection{Generalized Intensity-Hue-Saturation (GIHS)}

In the HIS color space, intensity (I) is a measure of brightness, which is sometimes called luminance (L), Hue, (H) is the color, measured as the angle around a color wheel or color hexagon (Almoni, 2007), and Saturation is the amount of color, representing the orthogonal distance from intensity axis in the HIS color space (Gonzalez, 2002). Generalized IHS (GIHS) or Fast IHS (FIHS) is a unifying image fusion method in which the low resolution intensity component $\left(\mathrm{I}_{0}\right)$ in HIS space is replaced by a graylevel image with higher spatial resolution ( $\mathrm{I}_{\text {new }}$ or pan) and transformed back to the original RGB space with the original $\mathrm{H}$ and $\mathrm{S}$ components (Tu, 2001).

$$
\left[\begin{array}{c}
R_{\text {new }} \\
G_{\text {new }} \\
B_{\text {new }}
\end{array}\right]=\left[\begin{array}{ccc}
1 & -1 / \sqrt{2} & 1 / \sqrt{2} \\
1 & -1 / \sqrt{2} & -1 / \sqrt{2} \\
1 & \sqrt{2} & 0
\end{array}\right]\left[\begin{array}{c}
I_{\text {new }} \\
v 1_{0} \\
v 2_{0}
\end{array}\right]
$$

To develop a computationally efficient method without the coordinate transformation, (1) can be rewritten as

$$
\left[\begin{array}{l}
R_{\text {new }} \\
G_{\text {new }} \\
B_{\text {new }}
\end{array}\right]=\left[\begin{array}{ccc}
1 & -1 / \sqrt{2} & 1 / \sqrt{2} \\
1 & -1 / \sqrt{2} & -1 / \sqrt{2} \\
1 & \sqrt{2} & 0
\end{array}\right]\left[\begin{array}{c}
I_{0}+\left(I_{\text {new }}-I_{0}\right) \\
v 1_{0} \\
v 2_{0}
\end{array}\right]=\left[\begin{array}{l}
R_{0}+\delta \\
G_{0}+\delta \\
B_{0}+\delta
\end{array}\right]
$$

where $\delta=I_{\text {new }}-I_{0}$. So, the fused image $\left[R_{\text {new }}, G_{\text {new }}, B_{\text {new }}\right]^{T}$ can be easily obtained from the resized original image $\left[R_{0}, G_{0}, B_{0}\right]^{T}$, simply by addition. A merit of the method lies not only on its fast computing capability for fused images but also its ability to extend traditional three order transformations to arbitrary order. That is $\mathrm{F}_{\mathrm{i}}=\mathrm{M}_{\mathrm{i}}+\beta$, where $\mathrm{M}_{\mathrm{i}}$ denotes the resized hyperspectral (HS) or multispectral (MS) image of band i, $\beta=$ Pan $-I_{0}$, and $I_{0}=\left((1 / l) \sum_{i=1}^{l} M_{i}\right)$, 1 represents the order or the number of bands $(\mathrm{Tu}, 2001) . \mathrm{I}_{0}$ value can be computed as the band distances, their weighted (adjusted) average or simply their average same as the above mentioned, in WorldView-1(Tu, 2012). Using weighting coefficients on green and blue band (for IKONOS image) in an attempt to minimize the difference between I and panchromatic image, leads to spectral adjusted IHS (SA-IHS) method.

\subsection{Brovey Transform (BT)}

In contrast to the IHS method, the BT method is a ratio fusion technique that preserves the relative spectral contribution of each pixel, but replaces its overall brightness by the high-resolution panchromatic image $(\mathrm{Tu}$, 2005). It is operated by

$$
\left[\begin{array}{l}
R_{B T} \\
G_{B T} \\
B_{B T}
\end{array}\right]=\frac{P a n}{I}\left[\begin{array}{l}
R \\
G \\
B
\end{array}\right]=\gamma_{B T}\left[\begin{array}{l}
R \\
G \\
B
\end{array}\right]
$$

And I, is the intensity of resized MS or HS image calculated as the above.

\subsection{Smoothing-Filter-Based Intensity Modulation (SFIM)}

SFIM is a BT-like approach, uses a smooth version of pan image $\left(\mathrm{P}_{\mathrm{L}}\right)$, in lieu of MS image intensity component as denominator of defined ratio $\left(\gamma_{B T}\right)$. Thereupon, this method is defined by

$$
\left[\begin{array}{l}
R_{B T} \\
G_{B T} \\
B_{B T}
\end{array}\right]=\frac{\operatorname{Pan}}{P_{L}}\left[\begin{array}{l}
R \\
G \\
B
\end{array}\right]
$$

where, $\mathrm{P}_{\mathrm{L}}$ is often obtained from a $7 \times 7$ mean filter. It is known that the spatial resolution can be improved by increasing the mask size of the low-pass filter in the SFIM method ( $\mathrm{Tu}, 2012)$.

\subsection{An Adjustable GIHS-BT-SFIM}

As it would be mentioned in the next part, IHS technique generates significant saturation compression, and the BT method suffers from saturation stretch. Motivated by this idea, an adjustable IHS-BT approach was used (Tu, 2005), then the SFIM method incorporates into the IHS-BT-SFIM approach $(\mathrm{Tu}, 2012)$ by the following equation.

$\left[\begin{array}{l}R_{\text {new }} \\ G_{\text {new }} \\ B_{\text {new }}\end{array}\right]=\frac{P}{I+k_{1} \cdot(\hat{P}-I)}\left[\begin{array}{l}R+k_{2} \cdot(\hat{P}-I) \\ G+k_{2} \cdot(\hat{P}-I) \\ B+k_{2} \cdot(\hat{P}-I)\end{array}\right] \quad \hat{P}=P$ or $P_{L}$

$\mathrm{k}_{\mathrm{i}}$ is the module selection parameters and defined as to be $0 \leq k_{i} \leq 1$.

\section{EDGE ADAPTIVE IHS}

An Edge adaptive procedure was introduced by Rahmani et al. to enforce fidelity away from the edges. They have used an edge detecting function, $\mathrm{h}(\mathrm{x})$ in the IHS fusion method in the manner of

$$
F_{i}=M_{i}+h(x)(P-I)
$$

Where, $F_{i}$ is the fused image and $M_{i}$ is the band $i$ of the MS image. In this case $\mathrm{h}(\mathrm{x})$ is equal to one on edges and equal to zero off edges. Edge adaptive method produces images with high spectral resolution while maintaining the highquality spatial resolution of the original IHS. 


\section{EDGE ADAPTIVE GIHS-BT-SFIM}

In many applications such as precision farming and urban classification, there's a need to have more precise information on edges because of the error occurrence is especially there. Using MS image data off edges and the minimum saturation on edges presents a good quality on spatial and spectral information for image based analysis purposes similar to classification. So, in this improved method edges are transferred from the GIHS-BT-SFIM image to the MS image. This approach extracts the edges from the pan-chromatic image; where there are edges the IHS-BT-SFIM product was imposed, otherwise, the MS image was used. In this case the fused multichannel image can be formed by the new formula,

$$
\begin{aligned}
& I^{\prime \prime}=\frac{P}{P+(I-P) \cdot h(x)+k 1 \cdot h(x) \cdot(\hat{P}-I)}(I+k 2 \cdot h(x) \cdot(\hat{P}-I)) \\
& \hat{P}=P \text { or } P_{L}
\end{aligned}
$$

Another approach, which uses panchromatic image on edges and GIHS-BT-SFIM off edges, can be introduced as equation (8),

$$
\begin{aligned}
& I^{\prime \prime}=\frac{P}{I+k 1 \cdot h(x) \cdot(\hat{P}-I)}(I+k 2 \cdot h(x) \cdot(\hat{P}-I)) \\
& \hat{P}=P \text { or } P_{L}
\end{aligned}
$$

The edges can be extracted using standard edge detection methods such as the canny detector (Canny, 1986). There is another edge detector suggested by Prona and Malik (1990) whereby the considerable results can be achieved in a fusion task which was introduced as the best in this study among other edge detectors in (Rahmani, et al. 2008),

$$
h(x)=\exp \left(-\frac{\lambda}{|\nabla P|^{4}+\varepsilon}\right) ; \lambda=10^{-9} \text { and } \varepsilon=10^{-10}
$$

(9) The best edge detector in this study was Roberts, as experienced.

\section{CLASS-BASED FUSION APPROACH}

Because of different distortion extent due to each class, it was interesting to test every method in a class-based manner. This part was just tested on urban image because of more distinct nature of the classes and only two different types of objects defined as urban and vegetation cover. A class based fusion was tested, which gives different coefficients for each method due to its class. It means that, two adjustable parameters, introduced in first part for defining combination of fusion methods, has changed in a LUT form and their best values were selected according to the fusion results assessment in each class. There were 16 miscellaneous cases which were tested and acquainted in the following part.

\section{RESULTS}

Two datasets have been tested in this study. First one was a high resolution image in an urban area and the other a medium resolution image of an agricultural field. Setting $\mathrm{k}_{\mathrm{i}}$ parameters of the GIHS-BT-SFIM, leads us to different fusion techniques and comparison for each method and their spectral and spatial quality was simply implemented. Spatial quality can be judged visually, but subtle color changes are more difficult to notice in this manner (Rahmani et al, 2010). In order to compare method results, spatial and spectral qualities by relying on both visual inspection and metric performance data were evaluated. Band-to-band correlation coefficient (CC), distortion extent, ERGAS, SAM, Q Index (Q-average), RMSE and RASE are the spectral metrics, and CC and average gradient are the spatial metrics for analyzing fusion methods fulfillment (Rahmani, 2008; Alparone, 2008; Zhang, 2008).

\subsection{Urban area}

The first study area is an urban region located in Shahriar, (Longitude: $51^{\circ} 3^{\prime}$, latitude: $35^{\circ} 39^{\prime}$ ) Tehran, Iran. The

\begin{tabular}{|c|c|c|c|c|c|c|c|c|c|c|}
\hline 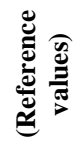 & 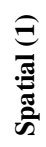 & 己َّ & $\underset{ت}{\leftarrow}$ & $\underset{U}{\sum_{0}^{\infty}}$ &  & $\vec{\Xi}$ & 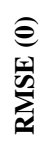 & 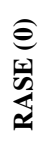 & 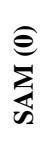 & $\begin{array}{l}\hat{\theta} \\
\hat{\sigma}\end{array}$ \\
\hline $\begin{array}{l}\text { IHS } \\
\hat{P}=I \\
k 1=k 2 \\
=1\end{array}$ & 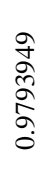 & 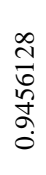 & $\begin{array}{l}\hat{n} \\
\infty \\
\infty \\
i n \\
n\end{array}$ & $\begin{array}{l}\infty \\
\stackrel{\Lambda}{0} \\
\text { ते } \\
\text { त̦ } \\
0\end{array}$ & $\begin{array}{l}\text { กิ } \\
\text { ลิ } \\
\text { ลิ }\end{array}$ &  & 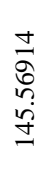 & 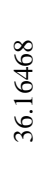 & $\begin{array}{l}\stackrel{n}{a} \\
\stackrel{3}{\Xi} \\
i\end{array}$ & 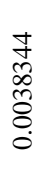 \\
\hline $\begin{array}{c}\begin{array}{c}\text { IHS- } \\
\text { BT }\end{array} \\
\hat{P}=I \\
k 1=k 2 \\
=0.5\end{array}$ & 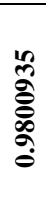 & 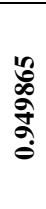 & 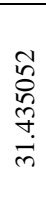 & $\begin{array}{l}\frac{9}{7} \\
\vdots \\
\stackrel{1}{0} \\
0\end{array}$ & 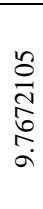 & 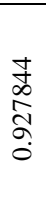 & 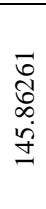 & 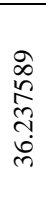 & 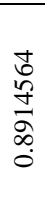 & 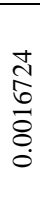 \\
\hline $\begin{array}{l}\quad \text { BT } \\
\hat{P}=I \\
k 1=k 2 \\
=0\end{array}$ & 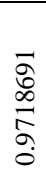 & 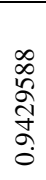 &  & 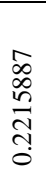 & $\begin{array}{l}\bar{n} \\
\hat{b} \\
\infty \\
\stackrel{0}{\sigma}\end{array}$ &  & 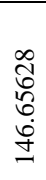 & \begin{tabular}{l}
$\stackrel{8}{8}$ \\
$\stackrel{+}{f}$ \\
\multirow{f}{*}{} \\
$\dot{n}$
\end{tabular} &  &  \\
\hline $\begin{array}{l}\text { BT- } \\
\text { SFIM } \\
\hat{P}=P_{L} \\
k 1=k 2 \\
=1\end{array}$ & $\begin{array}{l}0 \\
0 \\
+ \\
0 \\
\tilde{\sigma} \\
\vdots \\
0\end{array}$ & $\begin{array}{l}2 \\
\infty \\
\frac{\infty}{8} \\
\dot{\sigma}\end{array}$ & $\begin{array}{l}\hat{o} \\
\text { + } \\
\frac{n}{n} \\
\frac{n}{n}\end{array}$ & 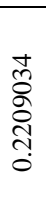 & $\begin{array}{l}\frac{n}{0} \\
\frac{a}{a} \\
\stackrel{a}{a}\end{array}$ & $\frac{\vec{a}}{\stackrel{a}{a}}$ & $\begin{array}{l}\vec{g} \\
\stackrel{7}{a} \\
\stackrel{g}{g}\end{array}$ & 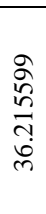 &  & 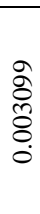 \\
\hline $\begin{array}{l}\text { SFIM } \\
\hat{P}=P_{L} \\
k 1=1 \\
k 2=0\end{array}$ & $\begin{array}{l}\stackrel{a}{N} \\
\stackrel{ }{\vec{N}} \\
\infty \\
0 \\
0\end{array}$ &  & $\begin{array}{l}\infty \\
\stackrel{0}{0} \\
\infty \\
\infty \\
\infty \\
\infty \\
\infty\end{array}$ & $\begin{array}{l}\hat{\sigma} \\
\stackrel{\circ}{\circ} \\
\text { ले } \\
\stackrel{0}{0}\end{array}$ &  & $\begin{array}{l}\frac{m}{a} \\
\frac{a}{5} \\
\vdots \\
a\end{array}$ & 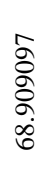 & $\frac{\vec{n}}{\stackrel{2}{\sigma}}$ &  & $\begin{array}{l}\exists \\
\equiv \\
\overline{8} \\
8 \\
0\end{array}$ \\
\hline
\end{tabular}
IKONOS image with four bands (Red, Green, Blue and Near infrared) and 3 meters spatial resolution in MS and one panchromatic band with 1 meter spatial resolution was used in this region (Figure 1). Results (Table 1) are 


\begin{tabular}{|c|c|c|c|c|c|c|c|c|c|c|}
\hline $\begin{array}{c}\text { Edge } \\
+ \text { IHS }\end{array}$ & $\begin{array}{l}n \\
\\
0 \\
\infty \\
0 \\
0\end{array}$ & $\begin{array}{l}0 \\
\vdots \\
10 \\
\\
\infty \\
0 \\
0\end{array}$ & 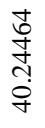 &  & $\begin{array}{l}\hat{0} \\
\hat{0} \\
+ \\
\infty \\
\infty\end{array}$ & $\begin{array}{l}\tilde{N} \\
\stackrel{2}{2} \\
\grave{o}\end{array}$ & $\begin{array}{l}\text { ț } \\
\stackrel{5}{a} \\
\stackrel{m}{2}\end{array}$ & 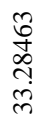 & 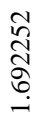 & 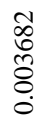 \\
\hline $\begin{array}{c}\text { Edge } \\
+ \\
\text { IHS- } \\
\text { BT } \\
(\mathbf{k}= \\
\mathbf{0 . 5}) \\
\end{array}$ & $\begin{array}{l}\overleftarrow{J} \\
0 \\
0 \\
0 \\
0 \\
0\end{array}$ & $\mid \begin{array}{c}0 \\
0 \\
1 \\
\infty \\
0 \\
0\end{array}$ & $\frac{m}{\mathfrak{o}}$ &  & 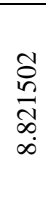 & $\begin{array}{l}\text { J } \\
\vdots \\
\vdots \\
0 \\
0\end{array}$ & 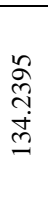 & $\begin{array}{l}\stackrel{\infty}{\circ} \\
\stackrel{+}{m} \\
m \\
m\end{array}$ & \begin{tabular}{l}
$\vec{\infty}$ \\
2 \\
\multirow{2}{+}{} \\
$\vdots$
\end{tabular} & $\frac{9}{8}$ \\
\hline $\begin{array}{l}\text { Edge } \\
+ \text { BT }\end{array}$ &  & $\begin{array}{l}2 \\
\dot{0} \\
\infty \\
0 \\
\infty \\
0\end{array}$ & 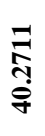 & 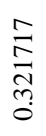 &  & $\begin{array}{l}\text { बे } \\
\frac{\partial}{\sigma}\end{array}$ & $\begin{array}{l}\stackrel{0}{\sigma} \\
\text { aे } \\
\dot{m}\end{array}$ & $\begin{array}{l}m \\
m \\
n \\
m \\
m\end{array}$ & 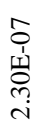 & 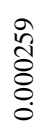 \\
\hline $\begin{array}{c}\text { Edge } \\
\text { + BT- } \\
\text { SFIM }\end{array}$ & \begin{tabular}{l}
$\frac{\sigma}{+}$ \\
\multirow{5}{*}{} \\
0 \\
0
\end{tabular} & $\mid \begin{array}{c}\infty \\
0 \\
0 \\
\infty \\
\infty \\
\infty \\
0\end{array}$ & 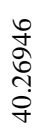 &  & $\begin{array}{l}\mathcal{N} \\
\underset{\infty}{F} \\
\infty \\
\infty \\
\infty\end{array}$ & $\begin{array}{l}\underset{J}{J} \\
\stackrel{\Xi}{\sigma} \\
0\end{array}$ & $\begin{array}{l}0 \\
\infty \\
\stackrel{+}{+} \\
\dot{m} \\
=\end{array}$ & $\begin{array}{l}\stackrel{+}{m} \\
m \\
m \\
m\end{array}$ & $\begin{array}{l}8 \\
+ \\
+ \\
+1 \\
+ \\
\text { m }\end{array}$ & $\begin{array}{l}\text { đo } \\
\text { ¿ै } \\
8 \\
0\end{array}$ \\
\hline $\begin{array}{c}\text { Edge } \\
+ \\
\text { SFIM }\end{array}$ & 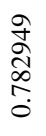 &  & 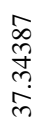 & 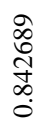 & $\begin{array}{l}\vec{y} \\
\stackrel{\text { J }}{ \pm} \\
\dot{+}\end{array}$ & $\begin{array}{l}\frac{a}{8} \\
\frac{1}{5} \\
\vdots \\
0\end{array}$ & \begin{tabular}{l}
$\hat{a}$ \\
\multirow{0}{0}{} \\
$\dot{b}$ \\
$\dot{0}$
\end{tabular} & 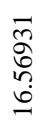 & 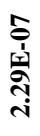 & $\begin{array}{l}1 \\
\\
8 \\
0 \\
0\end{array}$ \\
\hline $\begin{array}{c}\text { Edge: } \\
\text { HIS } \\
\text { +MS }\end{array}$ &  & $\begin{array}{l}\vec{\infty} \\
\infty \\
\stackrel{n}{n} \\
\tilde{0}\end{array}$ &  & 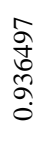 & 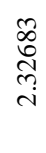 & 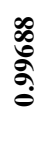 & $\frac{\overrightarrow{5}}{0}$ & 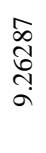 & 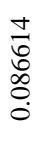 & 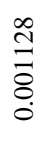 \\
\hline $\begin{array}{c}\text { Edge: } \\
\text { HIS } \\
\text { BT } \\
+ \text { MS }\end{array}$ & 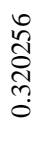 & 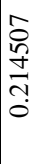 & 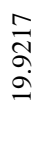 & $\begin{array}{l}\hat{\delta} \\
\text { ò } \\
\hat{\sigma}\end{array}$ & 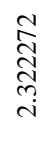 & $\begin{array}{l}\frac{N}{J} \\
\stackrel{\circ}{\sigma} \\
\delta\end{array}$ & $\begin{array}{l}\vec{\Xi} \\
\stackrel{\Xi}{0} \\
\stackrel{0}{0}\end{array}$ & 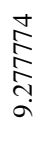 & 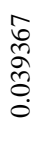 & 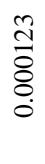 \\
\hline $\begin{array}{c}\text { Edge: } \\
\text { BT } \\
+ \text { MS }\end{array}$ & 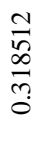 & 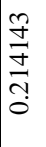 & 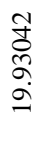 & 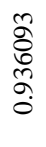 &  & $\begin{array}{l}\hat{a} \\
\text { Jे } \\
\text { à }\end{array}$ & $\frac{n}{n}$ & $\begin{array}{l}n \\
\infty \\
\infty \\
\infty \\
\tilde{+} \\
a\end{array}$ & $\begin{array}{l}5 \\
\text { 1 } \\
\text { wn } \\
\text { r. } \\
\text { ci }\end{array}$ & $\begin{array}{l}n \\
0 \\
1 \\
1 \\
0 \\
0 \\
-\end{array}$ \\
\hline $\begin{array}{c}\text { Edge: } \\
\text { BT- } \\
\text { SFIM } \\
+ \text { MS }\end{array}$ & $\frac{\infty}{\stackrel{2}{\sigma}}$ & 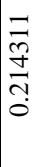 & 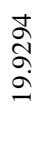 & 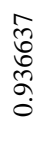 & 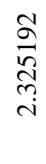 &  & $\frac{\infty}{\stackrel{\infty}{*}}$ & $\begin{array}{l}\text { ठे } \\
\text { مे } \\
\text { مे }\end{array}$ & 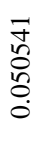 & $\begin{array}{l}\frac{\infty}{8} \\
\stackrel{8}{8} \\
0 \\
0\end{array}$ \\
\hline $\begin{array}{c}\text { Edge: } \\
\text { SFIM } \\
\text { +MS }\end{array}$ & 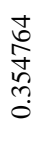 &  & 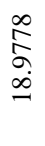 & 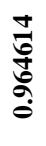 & $\begin{array}{l}\overline{7} \\
\text { กิ } \\
\text { ñ } \\
\text { ? }\end{array}$ & 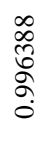 & 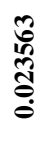 &  & 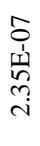 & 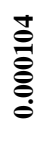 \\
\hline
\end{tabular}

Table 1: Assessing the fusion methods in an urban region

It is clear that edge adaptive method has improved spectral information in all procedures. Fusion results are provided in figure 1 .

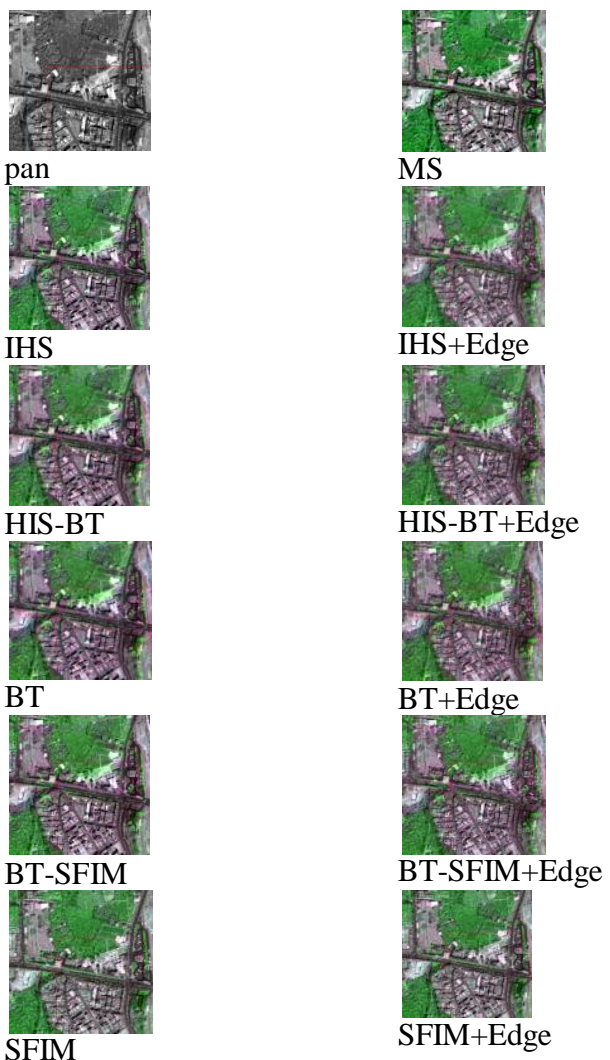

Figure 1:Results for Urban area

\subsection{Agricultural fields}

The area of interest deals with agricultural fields located around the village of Biddinghuizen in Flevoland, the Netherlands, which is characterized by the cultivation of arable crops (Abkar, 1994). This area represented a typical agricultural region in the Netherlands. The main crops are grass, potatoes, cereals, sugar-beets, beans, peas, and onions. The three spectral bands 3, 4, and 5 of the Landsat Thematic Mapper (TM) image that was acquired on 7 July 1987 has been used in this study. Results on this region are in (Table 2 and figure 2).

\begin{tabular}{|c|c|c|c|c|c|c|c|c|c|c|}
\hline 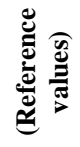 & 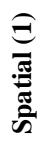 & 氮 & 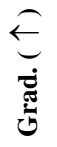 & 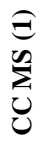 & 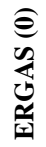 & $\begin{array}{l}\approx \\
\sigma\end{array}$ & 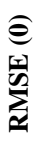 & 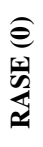 & 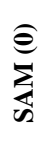 & $\begin{array}{l}\hat{\varepsilon} \\
\hat{\sigma}\end{array}$ \\
\hline $\begin{array}{l}\text { IHS } \\
\hat{P}=I \\
k 1=k 2 \\
=1\end{array}$ & 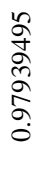 & 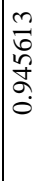 & 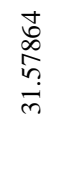 & 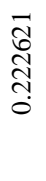 & $\begin{array}{l}\tilde{N} \\
\frac{\hat{\sigma}}{\sigma} \\
\text { à }\end{array}$ & 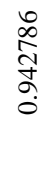 & 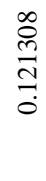 & 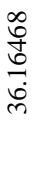 & 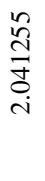 & $\begin{array}{l}\tilde{J} \\
\infty \\
\infty \\
\tilde{\delta} \\
0 \\
0\end{array}$ \\
\hline $\begin{array}{c}\begin{array}{c}\text { IHS- } \\
\text { BT }\end{array} \\
\hat{P}=I \\
k 1=k 2 \\
=0.5\end{array}$ & 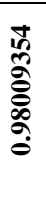 & $\mid \begin{array}{l}0 \\
0 \\
\infty \\
o \\
a \\
0 \\
0\end{array}$ & 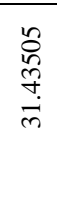 & 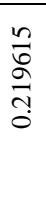 & $\begin{array}{l}\vec{\nabla} \\
\hat{\sigma} \\
\stackrel{\sigma}{a}\end{array}$ & 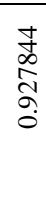 & $\frac{N}{n}$ & 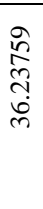 & 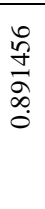 & 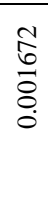 \\
\hline
\end{tabular}




\begin{tabular}{|c|c|c|c|c|c|c|c|c|c|c|}
\hline \begin{tabular}{l}
\multicolumn{1}{c}{ BT } \\
$\hat{P}=I$ \\
$k 1=k 2$ \\
$=0$
\end{tabular} & 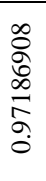 & 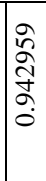 & 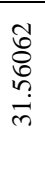 & $\begin{array}{l}\stackrel{2}{\infty} \\
\stackrel{n}{N} \\
\stackrel{\text { }}{0}\end{array}$ & $\begin{array}{l}\overline{\tilde{\sigma}} \\
\infty \\
\infty \\
\stackrel{\sigma}{0}\end{array}$ & $\begin{array}{l}\tilde{2} \\
\hat{\alpha} \\
\infty \\
0\end{array}$ & $\begin{array}{l}\underset{\mathbb{N}}{\mathbb{N}} \\
\stackrel{\text { I. }}{0} \\
\end{array}$ & 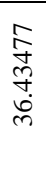 & 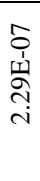 & 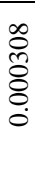 \\
\hline $\begin{array}{l}\text { BT- } \\
\text { SFIM } \\
\hat{P}=P_{L} \\
k 1=k 2 \\
=1\end{array}$ & 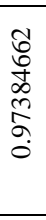 & 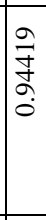 & $\frac{\bar{J}}{\bar{a}}$ & 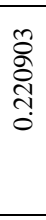 & $\begin{array}{l}\frac{n}{0} \\
\bar{a} \\
a \\
a\end{array}$ &  & $\frac{\stackrel{\infty}{+}}{\stackrel{+}{J}}$ & $\begin{array}{l}\stackrel{0}{n} \\
\text { ñ. } \\
\text { లn }\end{array}$ & 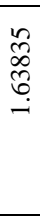 & 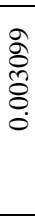 \\
\hline $\begin{array}{l}\text { SFIM } \\
\hat{P}=P_{L} \\
k 1=1 \\
k 2=0\end{array}$ & 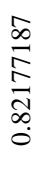 & 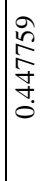 & $\begin{array}{l}\bar{m} \\
\infty \\
\tilde{\infty} \\
\infty \\
\infty \\
\infty\end{array}$ & $\begin{array}{l}\hat{\infty} \\
\text { ले } \\
\text { ल) } \\
\dot{0}\end{array}$ & 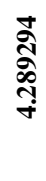 & $\begin{array}{l}\bar{\sigma} \\
\frac{1}{5} \\
\hat{0}\end{array}$ & 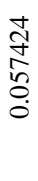 & $\begin{array}{l}\stackrel{n}{\Omega} \\
\Xi \\
\Xi\end{array}$ & 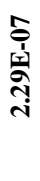 & $\begin{array}{l}\Xi \\
\bar{\theta} \\
\stackrel{8}{0}\end{array}$ \\
\hline $\begin{array}{c}\text { Edge } \\
+ \text { IHS }\end{array}$ & $\begin{array}{l}\hat{b} \\
\infty \\
\tilde{\infty} \\
0 \\
o \\
\stackrel{0}{0}\end{array}$ & 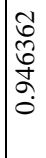 & $\frac{\text { No }}{\frac{N}{6}}$ & 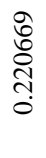 & $\begin{array}{l}\bar{\delta} \\
\text { ర్ర } \\
\infty \\
\infty \\
\sigma\end{array}$ & $\frac{m}{\tilde{N}}$ & $\frac{\infty}{\stackrel{\infty}{+}}$ &  & 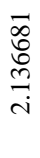 & $\begin{array}{l}\overrightarrow{7} \\
\stackrel{m}{8} \\
\stackrel{0}{0}\end{array}$ \\
\hline $\begin{array}{c}\text { Edge } \\
+ \\
\text { IHS- } \\
\text { BT } \\
(\mathbf{k}= \\
0.5)\end{array}$ & 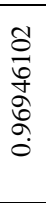 & 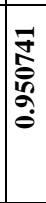 & $\begin{array}{l}\infty \\
\text { ñ } \\
\text { nn } \\
\text { n. }\end{array}$ & $\begin{array}{l}\hat{\circ} \\
\stackrel{n}{\sim} \\
\stackrel{\sim}{0}\end{array}$ &  &  & $\frac{⿱}{\stackrel{D}{二}}$ & 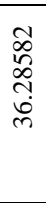 & 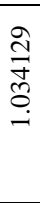 &  \\
\hline $\begin{array}{l}\text { Edge } \\
+ \text { BT }\end{array}$ &  & 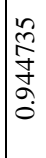 & $\begin{array}{l}\vec{\sigma} \\
\frac{m}{b} \\
\frac{m}{m}\end{array}$ & 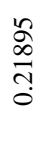 & 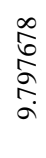 & $\begin{array}{l}\stackrel{m}{\infty} \\
\infty \\
\infty \\
\infty \\
0\end{array}$ & $\begin{array}{l}\stackrel{+}{D} \\
\stackrel{N}{N} \\
\stackrel{0}{0}\end{array}$ & 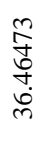 & $\begin{array}{l}\stackrel{+}{1} \\
\infty \\
0 \\
0\end{array}$ & $\begin{array}{l}\stackrel{0}{0} \\
\stackrel{8}{8} \\
\text { } \\
0\end{array}$ \\
\hline $\begin{array}{c}\text { Edge } \\
+ \text { BT- } \\
\text { SFIM }\end{array}$ & 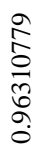 & 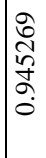 & $\begin{array}{l}n \\
\infty \\
\text { ô } \\
\infty \\
0 \\
\text { m }\end{array}$ & 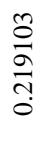 & $\begin{array}{l}\hat{n} \\
\stackrel{\infty}{\circ} \\
\dot{\sigma}\end{array}$ & 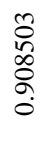 & 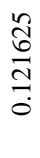 & 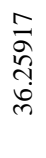 & 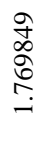 & 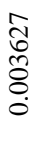 \\
\hline $\begin{array}{c}\text { Edge } \\
+ \\
\text { SFIM }\end{array}$ & 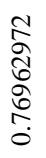 & $\begin{array}{l}0 \\
0 \\
\infty \\
0 \\
0 \\
+ \\
0\end{array}$ & 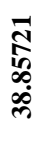 & $\begin{array}{l}n \\
n \\
o \\
\infty \\
0 \\
0\end{array}$ & 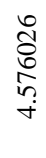 & 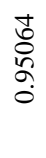 & $\begin{array}{l}\text { ते } \\
\text { } \\
\overline{0} \\
\stackrel{0}{0}\end{array}$ &  & $\begin{array}{l}\text { J } \\
\text { N } \\
\infty \\
0\end{array}$ & $\begin{array}{l}\frac{0}{\hat{s}} \\
\frac{8}{8} \\
0 \\
0\end{array}$ \\
\hline $\begin{array}{c}\text { Edge: } \\
\text { HIS } \\
\text { +MS }\end{array}$ & $\begin{array}{l}\stackrel{0}{\infty} \\
\infty \\
\stackrel{\infty}{0} \\
\stackrel{0}{0}\end{array}$ & 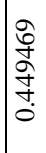 & $\begin{array}{l}\bar{\sigma} \\
6 \\
\frac{0}{\infty} \\
\infty\end{array}$ & $\begin{array}{l}\stackrel{n}{N} \\
\stackrel{N}{J} \\
\stackrel{0}{0}\end{array}$ & 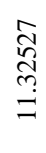 &  & \begin{tabular}{l}
$n$ \\
$n$ \\
\multirow{2}{0}{} \\
0 \\
0
\end{tabular} & 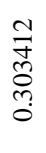 & $\begin{array}{l}\frac{n}{n} \\
\frac{n}{n} \\
n\end{array}$ & $\begin{array}{l}\infty \\
: \\
0 \\
\stackrel{0}{0} \\
\stackrel{0}{0}\end{array}$ \\
\hline $\begin{array}{c}\text { Edge: } \\
\text { HIS } \\
\text { BT } \\
+ \text { MS }\end{array}$ & 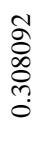 & 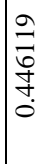 & \begin{tabular}{l}
\multirow{2}{0}{} \\
$\stackrel{n}{\infty}$ \\
$\infty$
\end{tabular} & $\begin{array}{l}\stackrel{0}{n} \\
\stackrel{+}{0} \\
0\end{array}$ & $\begin{array}{l}n \\
n \\
n \\
0\end{array}$ & 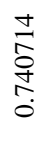 & 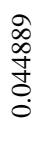 & 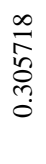 & 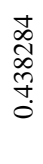 & 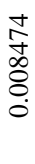 \\
\hline $\begin{array}{c}\text { Edge: } \\
\text { BT } \\
\text { +MS }\end{array}$ & 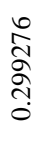 & 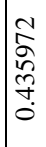 & $\begin{array}{l}0 \\
2 \\
\stackrel{+}{+} \\
\frac{\infty}{\infty}\end{array}$ & 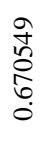 & $\begin{array}{l}0 \\
\delta \\
\infty \\
\infty \\
\infty\end{array}$ & $\frac{\substack{\infty \\
\stackrel{n}{n} \\
\stackrel{0}{0}}}{0}$ & 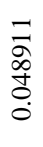 & $\underset{m}{\stackrel{7}{m}}$ & 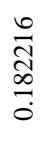 & $\begin{array}{l}\bar{\sigma} \\
\tilde{8} \\
0 \\
0\end{array}$ \\
\hline
\end{tabular}

\begin{tabular}{|c|c|c|c|c|c|c|c|c|c|c|}
\hline $\begin{array}{c}\text { Edge: } \\
\text { BT- } \\
\text { SFIM } \\
\text { +MS }\end{array}$ & 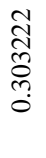 & $\begin{array}{l}\text { Oे } \\
\text { O̊ } \\
\text { I } \\
\text { J. } \\
0 \\
0\end{array}$ & 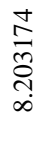 & 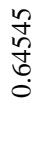 & 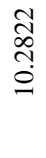 & 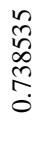 & 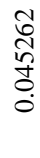 & $\begin{array}{l}\infty \\
n \\
\infty \\
0 \\
0 \\
0\end{array}$ & 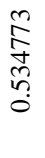 & 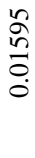 \\
\hline $\begin{array}{c}\text { Edge: } \\
\text { SFIM } \\
\text { +MS }\end{array}$ & 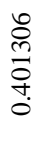 & $\begin{array}{l}0 \\
n \\
n \\
n \\
n \\
0\end{array}$ & $\begin{array}{l}0 \\
8 \\
8 \\
8 \\
0 \\
0\end{array}$ & 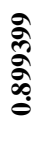 & $\begin{array}{l}\vec{d} \\
\infty \\
\infty \\
\infty \\
m \\
m\end{array}$ & $\frac{\substack{0 \\
+}}{\stackrel{+}{7}}$ & $\frac{\pi}{\frac{1}{0}}$ & $\frac{9}{\mathrm{~d}}$ & 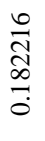 & $\begin{array}{l}= \\
\bar{n} \\
8 \\
0\end{array}$ \\
\hline
\end{tabular}

Table 2: Assessing the fusion methods in an agricultural field

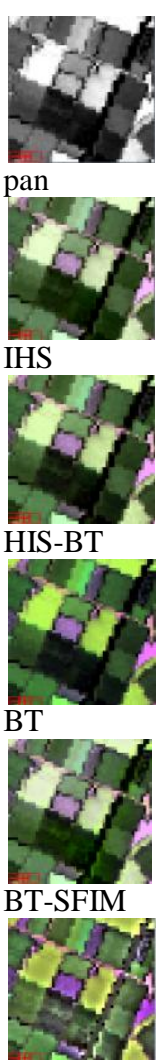

SFIM

Figure 2: Results for Agricultural field

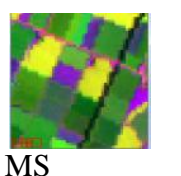

MS

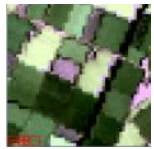

IHS+Edge

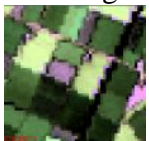

HIS-BT+Edge

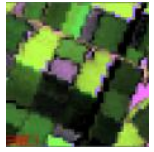

BT+Edge

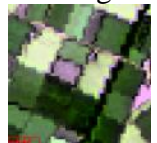

BT-SFIM+Edge

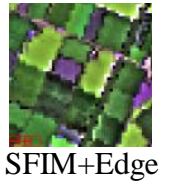

SFIM+Edge

\subsection{Class-Based Results (LUT-form)}

In this part, just the outputs are presented for each group of modulation parameters. It is worth knowing that class part results are defined due to some ROIs for each class, because of the memory limitation for processing the whole image and it may have some effects on the last results. Look up table for these parameters are here (Table 3).

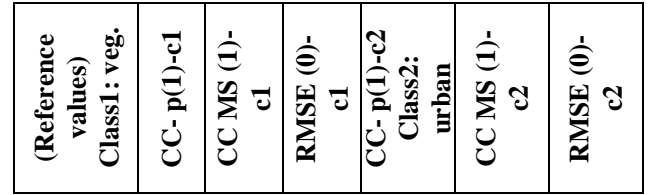




\begin{tabular}{|c|c|c|c|c|c|c|}
\hline $\begin{array}{l}\mathrm{K} 1=0.2 \\
\mathrm{~K} 2=0.2\end{array}$ & $\begin{array}{l}\hat{N} \\
\infty \\
\widetilde{\sigma} \\
0\end{array}$ & $\begin{array}{l}\text { to } \\
\text { సे } \\
\stackrel{5}{0}\end{array}$ & $\begin{array}{l}\infty \\
\infty \\
\infty \\
\varnothing \\
\delta \\
\dot{+}\end{array}$ & $\begin{array}{l}\tilde{\delta} \\
0 \\
o \\
o\end{array}$ & $\begin{array}{l}\frac{\tilde{f}}{\mathcal{J}} \\
\stackrel{J}{0}\end{array}$ & $\begin{array}{l}\text { के } \\
\stackrel{+}{0} \\
\infty \\
\stackrel{2}{n}\end{array}$ \\
\hline $\begin{array}{l}K 1=0.2 \\
K 2=0.4\end{array}$ & $\begin{array}{l}\vec{n} \\
\tilde{n} \\
a \\
o \\
0\end{array}$ & $\begin{array}{l}\text { t. } \\
\text { مे } \\
0 \\
0\end{array}$ & $\begin{array}{l}\frac{\infty}{\sigma} \\
\stackrel{\sigma}{\dot{n}}\end{array}$ & $\begin{array}{l}n \\
\infty \\
0 \\
0 \\
o \\
o\end{array}$ & $\begin{array}{l}\stackrel{2}{\hat{O}} \\
\stackrel{0}{0} \\
0\end{array}$ & $\begin{array}{l}\hat{f} \\
\dot{f} \\
\infty \\
\infty \\
\sigma \\
\sigma\end{array}$ \\
\hline $\begin{array}{l}K 1=0.2 \\
K 2=0.6\end{array}$ & 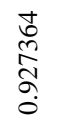 & $\begin{array}{l}\vec{b} \\
\text { v } \\
o \\
0 \\
0\end{array}$ & 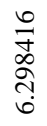 & \begin{tabular}{l}
\multirow{2}{0}{} \\
n. \\
$o$
\end{tabular} & $\begin{array}{l}\hat{a} \\
\infty \\
\stackrel{0}{0} \\
0\end{array}$ &  \\
\hline $\begin{array}{l}\mathrm{K} 1=0.2 \\
\mathrm{~K} 2=0.8\end{array}$ & 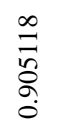 & 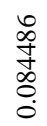 & 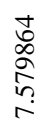 & 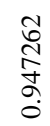 & $\begin{array}{l}\tilde{\delta} \\
0 \\
0 \\
0 \\
0\end{array}$ & $\begin{array}{l}\text { बे } \\
\text { oे } \\
\text { fे }\end{array}$ \\
\hline $\begin{array}{l}K 1=0.4 \\
K 2=0.2\end{array}$ & $\begin{array}{l}\overrightarrow{\tilde{\sigma}} \\
\stackrel{2}{\alpha} \\
\stackrel{0}{0}\end{array}$ & 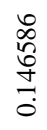 & $\begin{array}{c}\frac{\partial}{b} \\
\stackrel{m}{m} \\
\dot{m}\end{array}$ & $\begin{array}{l}\frac{m}{m} \\
\frac{\tilde{f}}{\alpha} \\
o\end{array}$ & $\begin{array}{l}\bar{m} \\
\stackrel{0}{0} \\
\stackrel{n}{0}\end{array}$ & 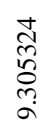 \\
\hline $\begin{array}{l}K 1=0.4 \\
K 2=0.4\end{array}$ &  & $\begin{array}{l}\vec{\Omega} \\
\stackrel{0}{0} \\
\stackrel{0}{0}\end{array}$ & $\begin{array}{l}\text { D. } \\
0 \\
\stackrel{0}{0} \\
\dot{+}\end{array}$ & $\begin{array}{l}n \\
\text { ñ } \\
\text { o. } \\
o\end{array}$ & $\begin{array}{l}\stackrel{+}{0} \\
\stackrel{\tilde{g}}{+} \\
0\end{array}$ & 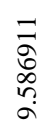 \\
\hline $\begin{array}{l}K 1=0.4 \\
K 2=0.6\end{array}$ & 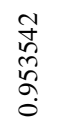 & 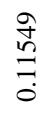 & 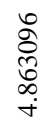 & $\begin{array}{l}\bar{\delta} \\
\hat{\sigma} \\
\text { o. }\end{array}$ & $\begin{array}{l}\vec{g} \\
\vec{m} \\
0\end{array}$ & 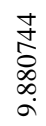 \\
\hline $\begin{array}{l}K 1=0.4 \\
K 2=0.8\end{array}$ & $\begin{array}{l}\text { Oे } \\
\text { Oे+ } \\
\text { Oे }\end{array}$ & 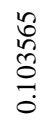 & $\begin{array}{l}n \\
\infty \\
\infty \\
\infty \\
\infty \\
i n\end{array}$ & $\begin{array}{l}n \\
\hat{2} \\
n \\
o \\
o\end{array}$ &  & $\begin{array}{l}\stackrel{0}{i} \\
\infty \\
\stackrel{\infty}{0}\end{array}$ \\
\hline $\begin{array}{l}K 1=0.6 \\
K 2=0.4\end{array}$ & $\begin{array}{l}\stackrel{\infty}{\infty} \\
\stackrel{2}{\hat{~}} \\
\hat{0}\end{array}$ &  & $\begin{array}{l}\text { ठু } \\
\text { } \\
\text { ते }\end{array}$ & $\begin{array}{l}\text { to } \\
\text { مे } \\
\text { a }\end{array}$ & $\begin{array}{l}\tilde{0} \\
\stackrel{0}{0} \\
0 \\
0\end{array}$ & $\begin{array}{l}\bar{\sigma} \\
\vec{\sigma} \\
\infty \\
\infty\end{array}$ \\
\hline $\begin{array}{l}K 1=0.6 \\
K 2=0.6\end{array}$ & $\frac{\tilde{a}}{\Xi}$ & 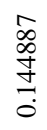 & 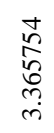 & $\begin{array}{l}\frac{m}{\infty} \\
\stackrel{\infty}{0} \\
0\end{array}$ & $\begin{array}{l}\hat{\sigma} \\
\hat{n} \\
\hat{n} \\
0\end{array}$ & 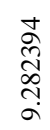 \\
\hline $\begin{array}{l}K 1=0.6 \\
K 2=0.8\end{array}$ & 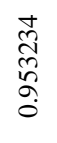 & 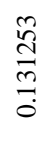 &  & $\begin{array}{l}\stackrel{\Xi}{\Xi} \\
\stackrel{\Xi}{0} \\
o\end{array}$ & $\begin{array}{l}\tilde{\sim} \\
\stackrel{\sim}{J} \\
\stackrel{J}{0}\end{array}$ & $\begin{array}{l}\text { శ్ } \\
\infty \\
\infty \\
n \\
n \\
\sigma\end{array}$ \\
\hline
\end{tabular}



Table 3: Class-based and modulation parameter effects on fusion results. c1; is for class one (vegetation cover) and c2; is for class2 (urban region).

\section{CONCLUSION}

In contrast with previous studies (Rahmani, 2010 \& Tu, 2012), which only contemplate the spectral metrics in their work; there's a need to have both spatial and spectral metrics because of the fusion goal; in this study, different spatial and spectral metrics have been presented and considering all is obvious that adding edge information form panchromatic image into GIHS_BT_SFIM product has not much improvement in various processes (Eq.8). Using edge information on edges and MS data off edges has shown spectral enhanced fused images according to metrics in various methods (Eq.7). Nonetheless, there is some spatial information loss. So, it is preferable to choose the best method of fusion due to task aims and image data. BT method in both images offers the most balanced results among all according to both spectral and spatial information, and SFIM has the most spectral information. Moreover, some of edge detectors' performance was tested, and results show the high dependence of edge adaptive method on the edge detector used.

It is clear that, method sort (the modulation parameter) is important in image fusion process and the fusion results are affected by the class type. So, choosing the best modulation parameters (methods) in a fusion task should be considered. It is recommended to study on class based results more, in the future.

\section{REFERENCES}

Abkar, A.A., 1994. Knowledge-based classification method for crop inventory using high spatial resolution data. A thesis, ITC, ENSCHEDE, The Netherlands. 
Alparone, L., Alazzi, B., Baronti, S., Garzelli, A., Nencini, F., Selva, M., 2008. Multispectral and panchromatic data fusion assessment without reference. In: Photogrammetric Engineering \& Remote Sensing.

Amolins, K., Zhang, Y., Dare, P., 2007. Wavelet based image fusion techniques- An introduction, review and comparison. In: Photogrammetry \& Remote Sensing.

Chue, H., Zhu, W., 2008. Fusion of IKONOS satellite imagery using HIS transform and local variation. In: IEEE Geoscience and Remote Sensing Letters.

Dou, W., Chen, Y., Li, X., Sui, D., 2007. A general framework for component substitution image fusion: an implementation using the fast image fusion method. In: Computers \& Geosciences.

Gonzalez, R., Woods, R., 2002, Digital Image Processing, Prentice Hall, pp. 282-302.

Li, Sh., Kwok, J., Wang, Y., 2002. Using the discrete wavelet frame transform to merge Landsat TM and SPOT panchromatic images. In: Information Fusion.

Li, Z., Jing, Z., Yang, X., Sun, Sh., 2005. Color transfer based remote sensing image fusion using non-separable wavelet frame transform. In: Pattern Recognition Letters.

Rahmani, Sh., Strait, M., Merkurjev, D., Moeller, M., Wittman, T., 2010. An adaptive HIS pan-sharpening method. In: IEEE Geoscience and Remote Sensing Letters.

Rahmani, Sh., Strait, M., Merkurjev, 2008. Evaluation of pan-sharpening methods.

Tu, T., Haung, Su, Sh., Shyu, H., P.S., Hung, 2001. A new look at HIS-like image fusion methods. In: Information Fusion.

Tu, T., Haung, P.S., Hung, Ch., Chang, Ch., 2004. A fast Intensity-Hue-Saturation fusion technique with spectral adjustment for IKONOS imagery. In: IEEE Geoscience and Remote Sensing Letters.

Tu, T., Lee, Y., Chang, Ch., Huang, P., 2005. Adjustable intensity-hue-saturation and brovey transform fusion technique for IKONOS/Quickbird imagery. In: Optical Engineering.

Tu, T., Hsu, Ch., Lee, Ch., 2012. An adjustable pansharpening approach for IKONOS/Quickbird/GeoEye1/Worldview-2 imagery. In: IEEE Journal of Selected Topics in applied Earth Observations and Remote Sensing.

Yang, X., Jing, Z., Liu, G., Hua, L., Ma, D., 2007. Fusion of multispectral and panchromatic images using fuzzy rule. In: Communications in Nonlinear Science and Numerical Simulation.

Zhang, Y., 2008. Methods for image fusion quality assessment-A Review, comparison and analysis. In: The
International Archives of the Photogrammetry, Remote Sensing and Spatial Information Sciences.

Zhang, Y., Hong, G., 2005. An HIS and wavelet integrated approach to improve pan-sharpening visual quality of natural colour IKONOS and QuickBird images. In: Information Fusion. 